\title{
Convective heat transfer in a rotating square channel with oblique cross section
}

\author{
S. Fann, W.-J. Yang
}

\begin{abstract}
A numerical study has been performed to study the fluid flow and convective heat transfer inside a rotating square isothermal channel with the channel cross-section having oblique angles to the rotational axis. The channel is subjected to a radial rotation. Computations are carried out for flows at $R e=500,1000$ and 2000 and range from the channel entrance to a flow distance of 300 and 600 times the hydraulic diameter, depending upon the Reynolds number. Results reveal the vortex flow structures, and consequently the heat transfer phenomena, are quite different from that of previous studies with zero oblique angle. The channel with 45 degree oblique angle yields the best overall heat transfer performance.
\end{abstract}

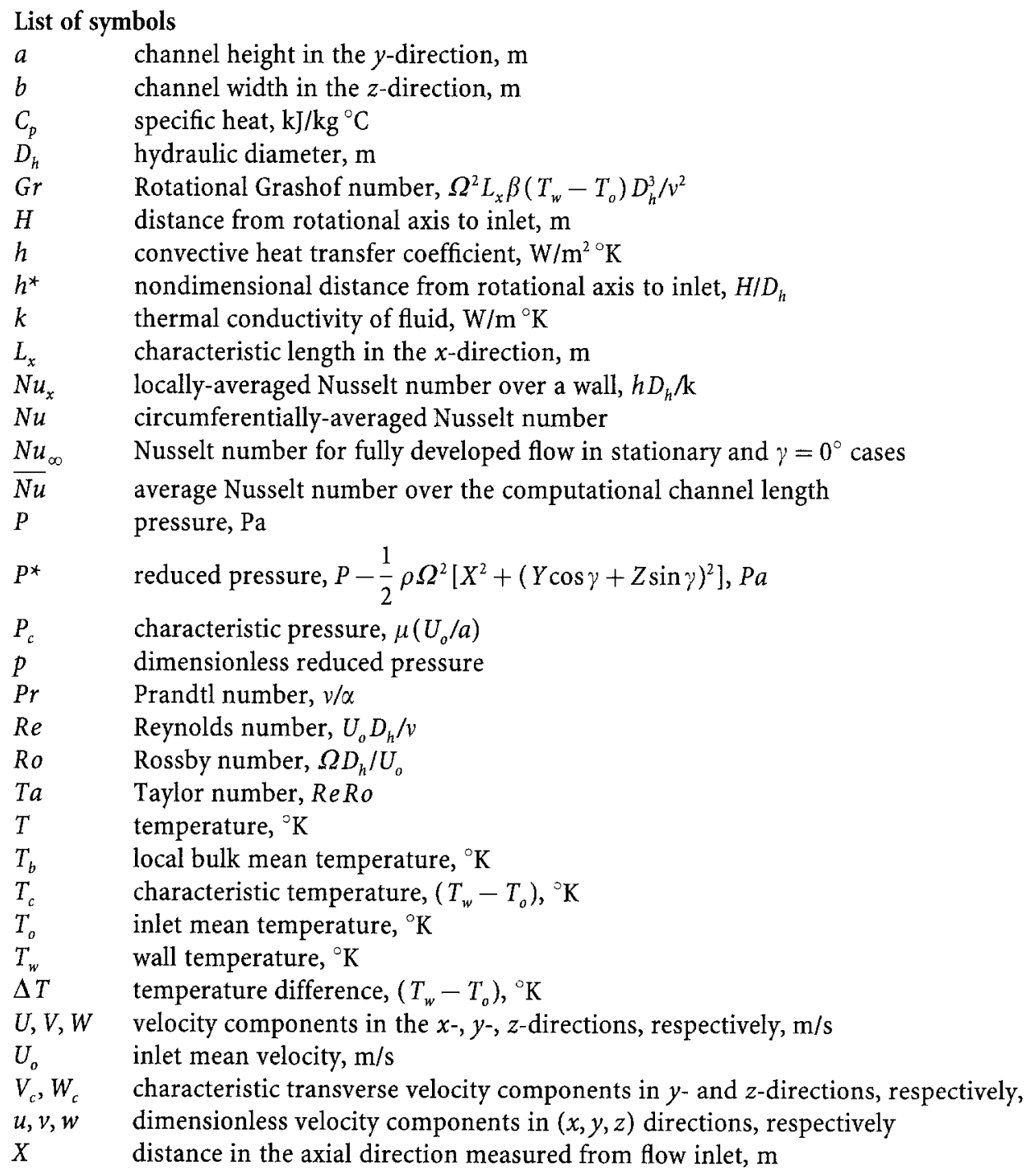

Communicated by Y. Jaluria, 28 February 1994

Department of Mechanical Engineering, and Applied Mechanics, The University of Michigan, Ann Arbor, Michigan 48109, USA 

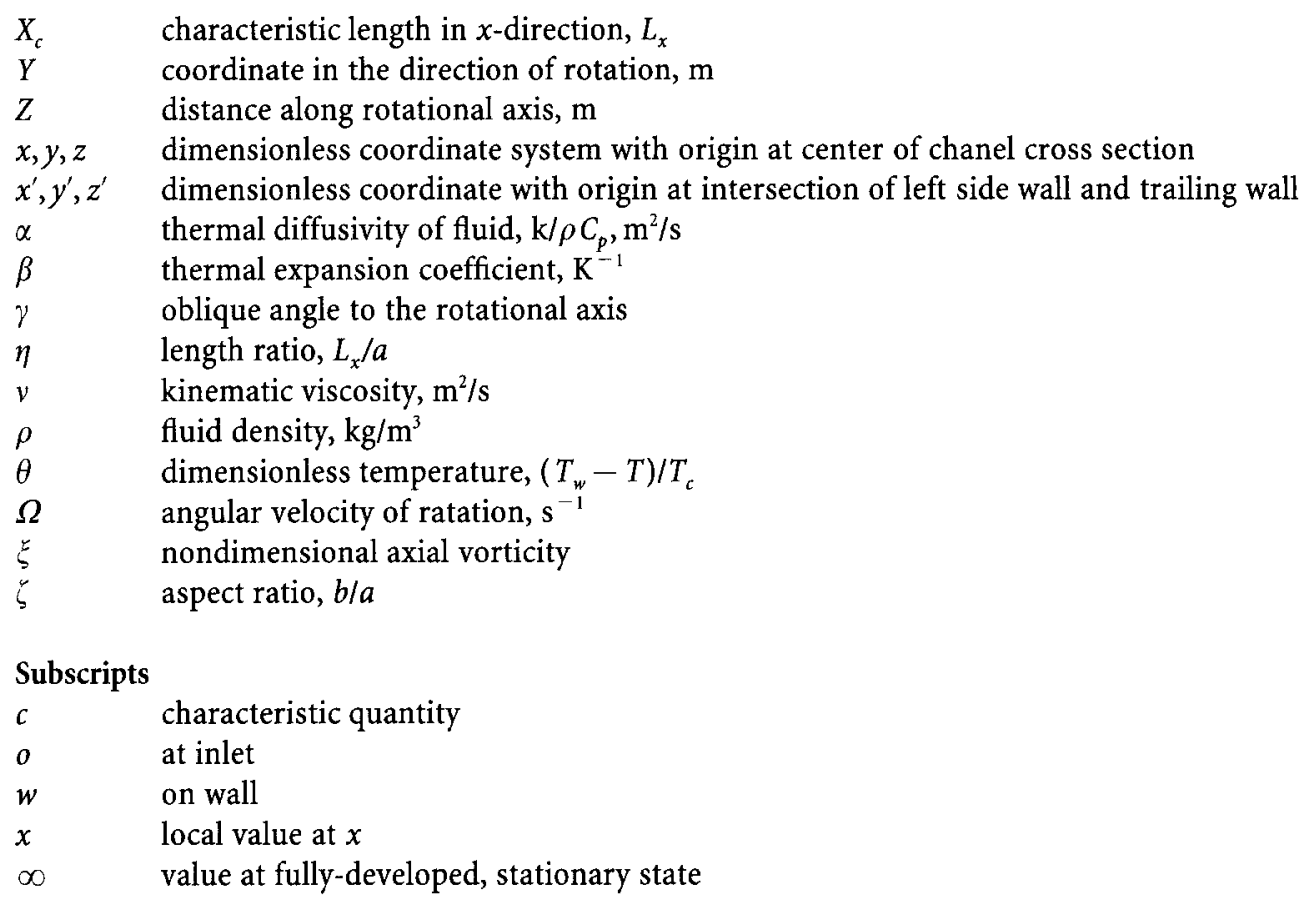

1

\section{Introduction}

Fluid flow and heat transfer in rotating systems, especially in radially rotating channels, have attracted considerable attention in the past several decades due to their important applications in industry. The problems involved in this topic include convective heat transfer, vortex flow evolution and heat transfer enhancement techniques. Under radial rotation, convective heat transfer has been confirmed to enhance with an increase in either the flow rate or the rotational speed, and the vortex flow variation along the channel has been also found to be related to the Reynolds number, Rossby number and heating conditions. However, most of the previous studies focus on the channel cross section with zero oblique angle, $\gamma=0$ (see Fig. 1). The present study makes an effort toward understanding the effects of inclined channel cross-section on vortex flow and convective heat transfer. Previous studies are briefly introduced in the following.

Hart (1971), Johnston et al. (1972), Smirnov and Yurkin (1983), Kuz'minskii et al. (1983), and Alfredsson and Persson (1989) experimentally studied flow instability phenomena in rotating channels of different aspect ratios, while Hart (1971), Lezius and Johnston (1976), Speziale (1982), Speziale and Thangam (1983), Kheshgi and Scriven $(1985)$, and Finlay $(1990,1992)$ investigated these phenomena theoretically. Hart (1971) found that in large aspect-ratio channels, there exist: (i) a double-vortex secondary flow at slow rotational speed, (ii) an instability in the form of longitudinal roll cells at intermediate rotational speed, and (iii) a restabilization of flow to a Taylor-Proudman regime at high rotational speed, where the axial velocity profile does not vary along the direction of the rotational axis. Johnston et al. (1972) also disclosed three stability-related phenomena in a channel of large aspect ratio: (i) a reduction (increase) in the rate of wall-layer streak bursting in locally stabilized (destabilized) wall layers; (ii) the

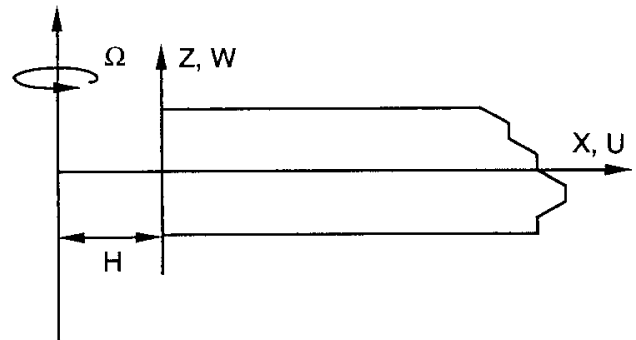

Side view

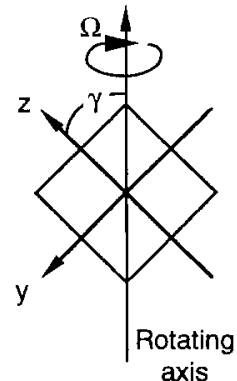

Front view

xis

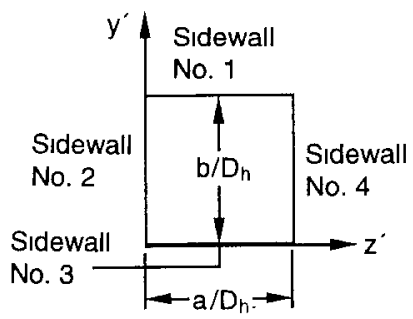

Fig. 1. Coordinate system on a rotating channel 
total suppression of transition to turbulence in a stabilized layer; (iii) the development of large-scale roll cells on the destabilized side of the channel due to the growth of a Taylor-Gortler vortex instability. Alfredsson and Persson (1989) observed that the primary instability takes the form of regularly spaced roll cells aligned in the flow direction, which may occur at as low as $R e=100$, which is almost two orders of magnitude lower than the critical Reynolds number for Tollmien-Schlichting waves in a stationary channel flow. The theoretically most unstable wavenumber was shown to agree the experimental data. At high Reynolds numbers, a secondary instability was revealed. A wavy-type disturbance was observed to develop into a large-scale 'turbulence' containing imbedded roll cells as the rotational speed is increased.

Smirnov and Yurkin (1983) studied the kinematics of water flow in a rotating square channel by means of both the hydrogen bubbles and dye injection methods. It was revealed that at $\operatorname{Re}>300$ in a restricted range of $R o$, the secondary vortex may appear, and that $R e<650$, the fully-developed secondary vortex remains steady during the rotational speeds tested. The irregular variation of vortices were also observed. Kuz'minskii et al. (1983) applied the two flow visualization methods to study flow instability phenomena on the trailing side of a rotating channel flow. The secondary vortices (near the trailing wall) were confirmed within one half of the channel section. In larger aspect-ratio channels $(\zeta=4$ and 7.2), the number of pair vortices tends to increase with $R o$ at constant $R e$ or to increase with $R e$ at constant $R o$. However, a small disturbance in mass flow through the channel may cause a change in the flow pattern. Lezius and Johnston performed a linear stability analysis on the onset of laminar roll cells in a rotating plane Poiseuille flow using a finite difference method. Results indicated the occurrence of the critical disturbance at $R e=88.53$ and $R o=0.5$. However, at a higher Reynolds number, the unstable conditions are initiated in the range of $0<R o<3$. These results predicted flow instability at a somewhat lower Reynolds number for a given Rossby number than that of Hart. Following the work of Speziale (1982), Speziale and Thangam (1983) conducted a numerical study on the secondary flow and roll-cell instability problem in a rotating channel with an aspect ratio of eight. They disclosed the roll-cell and Taylor-Proudman regimes that led to a substantial distortion of axial velocity profiles. The critical point was found at $R e=110$ and $R o=0.5$, higher than those predicted by Lezius and Johnston (1976). Kheshgi and Scriven (1985) studied the fully developed flow through a square channel with radial rotation using the penalty/Galerkin/finite element method. The Coriolis force acts on a fast-moving fluid in the channel core in the direction of the cross product of the mean fluid velocity and the channel's angular velocity. A flow structure with an ageostrophic, virtually inviscid core was uncovered when both the Coriolis and convective inertial forces are strong. This ageostrophic two-vortex structure becomes unstable when the strength of the convective inertial force increases past a critical value. The two-vortex (principal vortices) solutions metamorphoses into four-vortex (with secondary vortices) solutions at an imperfect bifurcation composed of a pair of turning points. However, the analysis postulated a symmetry condition about the channel centerline, which is not physically true.

Finlay $(1990,1992)$ applied a pesudo-spectral method to investigate the vortex evolution and the transition from laminar to turbulent flow in a rotating plane-Poiseuille flow. With an increase in the Reynolds number, two transitions were discovered by Finlay (1990) which cause a steady, periodic array of two-dimensional vortices (roll cells) to generate the traveling waves in the streamwise direction. Associated with the two transitions are two different type of wavy vortex flows: twisting and undulating. The undulating mode is very similar to a undulating vortex flow in curved channel and to a wavy Taylor vortex flow. At a low rotational speed, the twisting mode is similar to twisting vortices in a curved channel flow, but appears dissimilar at a higher rotational speed. Finlay (1992) found different combinations of fundamental frequency of wavy vortices as the flow rate is increased. As the Reynolds number is increased to a certain value, instantaneous flow fields show that the major flow feature is still streamwise-oriented vortices, but has lost the characteristics of a lower Reynolds number flow (in the absence of spanwise traveling).

Siegel (1985), Harasgama and Morris (1988), Hwang and Jen (1990), Morris and Ghavami-Nasr (1991), Jen et al. (1992), Jen and Lavine (1992), Fann and Yang (1992, 1993a, b), and Fann et al. (1992) investigated the convective heat transfer performance inside a rotating channels. Siegel (1985) numerically studied the buoyancy effect on a fully-developed laminar outward or inward flow in a radially rotating tube. It was concluded that in the radial inflow, the buoyancy tends to improve heat transfer, while in the radial outflow, the buoyancy tends to reduce heat transfer. Harasgama and Morris (1988) examined the influence of both the Coriolis-induced secondary flow and the centripetal buoyancy on the heat transfer characteristics in circular, triangular, and square coolant passages. It was confirmed that (i) an increase in the rotational speed or a reduction in the centripetal buoyancy causes the average heat transfer to augment in the radially outward flow but to diminish in the radially inward flow, and (ii) in the radially inward flow case, an increase in the centripetal buoyancy resulted in the local heat transfer to grow on the leading surface but to reduce on the trailing surface. Morris and Ghavami-Nasr (1991) experimentally studied the rotational effect on local heat transfer in a rectangular channel. It was found that the 
Coriolis-induced secondary flow enhances local heat transfer on the trailing surface. The opposite is true on the leading surface where a significant impediment to local heat transfer can occur. The centripetal buoyancy enhances heat transfer on both the leading and trailing surfaces as the wall-to-coolant temperature difference is increased with other governing parameters held constant.

Hwang and Jen (1990) theoretically investigated heat transfer in a hydrodynamically and thermally fully-developed flow in rotating isothermal ducts. A temperature distribution equation for a forced or combined convective flow in a non-rotating passage was employed to evaluate the temperature field in a rotating duct. It was revealed that a secondary vortex can exist in a certain range of $R e T a$ but may disappear at the same value of Re Ta. However, a symmetry condition about the channel centerline, which is not physically true, was imposed in calculation. Jen et al. (1992) utilized a formulation similar to the one used in the present study to investigate the simultaneously developing flow in a rotating isothermal square channel with the computation length ranging from the channel entrance to about $0.2-0.25 X /\left(D_{h} R e\right)$. A break down of secondary vortices was found for different Reynolds numbers at certain distances from the inlet. Jen and Lavine (1992) further studied the vortex flow evolution and convection heat transfer in the channels with different aspect ratios.

Fann and Yang (1992) and Fann et al. (1992) numerically studied the transport phenomena in the entrance region of radially rotating heated channels taking into account the effects of aspect ratio. Secondary vortices were found in the entrance regime in a very short distance downstream from the inlet with the vortex number varying depending upon the geometry, Reynolds number, Rossby number and location along the channel. Although the symmetry boundary condition at the channel centerline was employed, numerical experiments showed the same results as that without symmetry assumption within the short distance from the entrance. Fann and Yang (1983a) disclosed that the flow instability phenomena exist in a laminar flow through a rotating square channel by numerical smulations. Three different regimes were disclosed which characterize the vortex flow pattern: (i) At a low Reynolds number, only the principal vortex pair appear; (ii) at an intermediate Reynolds number, flow experiences three vortex patterns due to different rotational speed, namely, the principal vortex pair, principal vortices together with secondary vortices and restabilization to the principal vortices; and (iii) at higher Reynolds number, flow contains secondary vortices but without the restabilization as the rotational speed is increased. The breakdown to secondary vortices was also found. The effects of heating on the vortex flow evolution and convective heat transfer in a rotating square isothermal channel was investigated numerically by Fann and Yang (1993b).

The present work attempts to study the effect of oblique angle of the channel cross section, as well as the effects of increasing heating, on the vortex flow structure, and equivalently the convective heat transfer, inside a rotating square channel. The isothermal boundary condition is imposed. The velocityvorticity method (Ramakrishna et al. 1982) is employed in the formulation. The resulting parabolic equations are solved by means of the numerical method of Fann and Yang (1992) and fann et al. (1992).

\section{2}

\section{Theoretical analysis}

The physical system to be studied, as shown in Fig. 1, consists of a flow through a square channel rotating at a constant speed $\Omega$ about an axis normal to the longitudinal direction of the channel. The velocity components in the $x$-, $y$ - and $z$-directions are $u, v$, and $w$, respectively. In order to normalize the governing equations, the velocity, pressure, coordinate variables, and geometrical dimension are non-dimensionalized by dividing their respective characteristic quantitis as follows:

$$
\begin{gathered}
x=\frac{X}{L_{x}}, \quad y=\frac{Y}{a}, \quad z=\frac{Z}{b}, \quad u=\frac{U}{U_{o}}, \quad v=\frac{V}{V_{c}}, \quad w=\frac{W}{W_{c}}, \\
p=\frac{p^{*}}{p_{c}}, \quad \eta=\frac{L_{x}}{a}, \quad \zeta=\frac{b}{a}, \quad \xi=\frac{\Xi}{\xi} .
\end{gathered}
$$

Here, $P^{*}$ is the reduced pressure. The characteristic quantities, denoted by the subscript $c$, are defined in the nomenclature. The axial vorticity transport equation is introduced to eliminate the pressure gradient terms in both the $y$-and $z$-direction momentum equations. The non-dimensional axial vorticity can be expressed as

$\xi=\frac{\partial w}{\partial y}-\frac{1}{\zeta} \frac{\partial v}{\partial z}$.

Under the assumptions of constant physical properties (except fluid density), negligible viscous heating and axial heat conduction, the Boussinesq's approximation for an incompressible, steady, laminar flow 
are employed. Utilizing the formulation of velocity-vorticity method, the governing equations for the present study are derived in the followings (details in Fann and Yang (1992) and Fann et al. (1993)):

(a) $x$-direction equation:

$$
\begin{aligned}
u \frac{\partial u}{\partial x}+v \frac{\partial u}{\partial y}+\left(\frac{1}{\varsigma}\right) w \frac{\partial u}{\partial z}= & \left(\frac{-1}{R e}\right) \frac{\partial p}{\partial x}+2 \operatorname{Ro}(v \cos \gamma+w \sin \gamma) \\
& +\left(\frac{\eta G r}{R e^{2}}\right) \theta\left(x+\frac{h^{*}}{\eta}\right)+\left(\frac{\eta}{R e}\right) \frac{\partial^{2} u}{\partial y^{2}}+\left(\frac{\eta}{\varsigma^{2} R e}\right) \frac{\partial^{2} u}{\partial z^{2}}
\end{aligned}
$$

(b) Axial vorticity transport equation:

$$
\begin{aligned}
u \frac{\partial \xi}{\partial x}+v \frac{\partial \xi}{\partial y}+\left(\frac{1}{\zeta}\right) w \frac{\partial \xi}{\partial z}+\frac{\partial u}{\partial y} \frac{\partial w}{\partial x}-\left(\frac{1}{\zeta}\right) \frac{\partial u}{\partial z} \frac{\partial v}{\partial x}-\xi \frac{\partial u}{\partial x} \\
=2 \eta^{2} \operatorname{Ro}\left[\left(\frac{1}{\zeta}\right) \frac{\partial u}{\partial z} \cos \gamma-\frac{\partial u}{\partial y} \sin \gamma\right] \\
+\left(\frac{\eta G r}{\zeta R e^{2}}\right)\left\{\frac{\partial \theta}{\partial y}\left(y \cos \gamma \sin \gamma+\varsigma z \sin ^{2} \gamma\right)-\left(\frac{1}{\zeta}\right) \frac{\partial \theta}{\partial z}\left(y \cos ^{2} \gamma+\varsigma z \cos \gamma \sin \gamma\right)\right. \\
+\theta \cos \gamma \sin \gamma(1-\zeta)\}+\left(\frac{\eta}{R e}\right) \frac{\partial^{2} \xi}{\partial y^{2}}+\left(\frac{\eta}{\zeta^{2} R e}\right) \frac{\partial^{2} \xi}{\partial z^{2}}
\end{aligned}
$$

(c) Poisson equations for the transverse velocities:

$$
\begin{aligned}
& \frac{\partial^{2} v}{\partial y^{2}}+\frac{1}{\varsigma^{2}} \frac{\partial^{2} v}{\partial z^{2}}=-\frac{1}{\varsigma} \frac{\partial \zeta}{\partial z}-\frac{\partial^{2} u}{\partial x \partial y} \\
& \frac{\partial^{2} w}{\partial y^{2}}+\frac{1}{\varsigma^{2}} \frac{\partial^{2} w}{\partial z^{2}}=\frac{\partial \xi}{\partial y}-\frac{1}{\varsigma} \frac{\partial^{2} u}{\partial x \partial z}
\end{aligned}
$$

(d) Energy equation:

$$
u \frac{\partial \theta}{\partial x}+v \frac{\partial \theta}{\partial y}+\left(\frac{1}{\zeta}\right) w \frac{\partial \theta}{\partial z}=\left(\frac{\eta}{\operatorname{Pr} R e}\right) \frac{\partial^{2} \theta}{\partial y^{2}}+\left(\frac{\eta}{\zeta^{2} \operatorname{Pr} \operatorname{Re}}\right) \frac{\partial^{2} \theta}{\partial z^{2}}
$$

Here, the Poisson equations are derived from Eq. (1) and the continuity equation. The centripetal buoyancy force terms in Eqs. (2) and (3) are derived from the thermal expansion coefficient based on the Boussinesq's approximation. The aspect ratio $\zeta$ is kept in the equations for the future study. Because of a relatively strong inertia force in the axial direction, the second derivative (diffusion) terms in the $x$-direction may be neglected and these equations take a parabolic-elliptic form. This formulation is so-called the velocity-vorticity method.

The pressure term can be decoupled as

$p(x, y, z)=\bar{p}(x)+p^{\prime}(x, y, z)$

By making the parabolic assumption, $\partial p^{\prime} / \partial x \ll d \bar{p} / d x$, the axial pressure gradient is then given by

$$
-\frac{\partial p}{d x}=-\frac{\partial \bar{p}}{d x}=-f(x)
$$

where $f(x)$ is determined by the constant flow rate constraint. The similar numerical methods used in Fann and Yang (1992) and Fann et al. (1992) are employed to determine the axial velocity, vorticity and temperature. A staggered mesh network is employed in the numerical analysis. The scheme accuracy and the mesh dependence have been discussed in Fann and Yang. The axial velocity obtained must satisfy the continuity equation. Otherwise, a new axial pressure gradient is assumed and the computation is repeated for a new axial velocity, until the continuity equation is satisfied. In Eqs. (4) 
and (5), the second-order, centered difference method is employed to discretize the $y$-and $z$-derivatives, while the three-point backward difference method is used for the $x$ - derivatives. The boundary vorticity is numerically calculated based on the definition of Eq. (1). (see Fann and Yang (1992)).

The line iteration method with an under-relaxation factor is utilized in the computations of $u$ in order to improve the convergence speed. The point iteration method is employed in calculating $\xi, v$ and $w$, while the three-point forward (or backward) finite difference scheme is used in the calculations of the axial velocity gradients for the friction factor. The appropriate inlet and boundary conditions are:

at inlet: $u=1.0, \quad v=w=0, \quad p=0, \quad \xi=0$, and $\theta=1.0$

at walls: $\quad u=v=w=0, \quad \theta=0.0$.
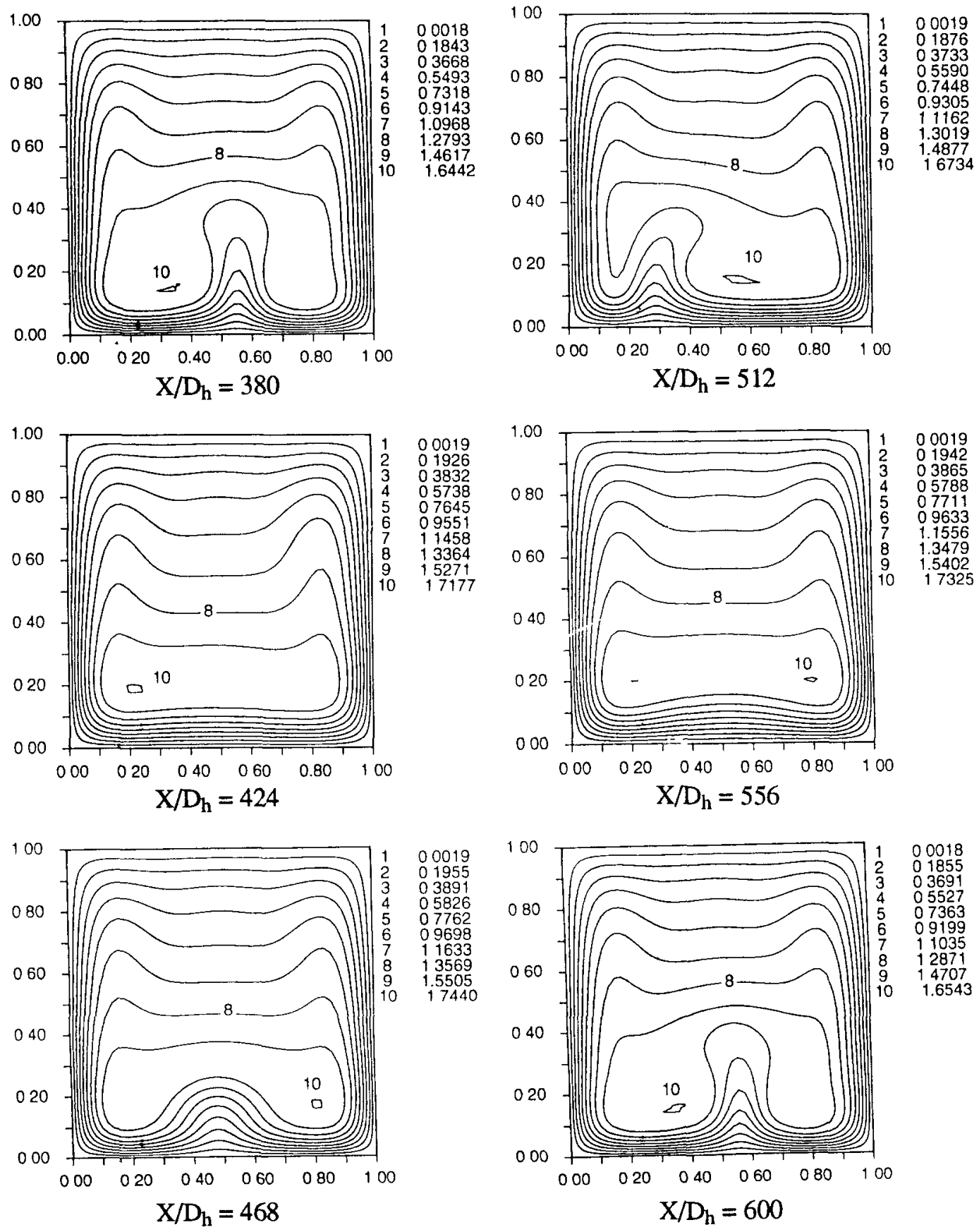

Fig. 2. Iso-lines of the axial flow velocity for flow at $R e=1000, R o=0.05, \Delta T=10$, and $\gamma=0^{\circ}$ 
The friction factor $f$ is defined as

$f=\frac{\mu\left(\frac{\overline{\partial U}}{\partial N}\right)_{\text {wall }}}{\frac{1}{2} \rho U_{o}^{2}}$

$f$ and $R e$ can be combined to give

$f R e=2\left(\overline{\frac{\partial u}{\partial n}}\right)_{\text {wall }}$
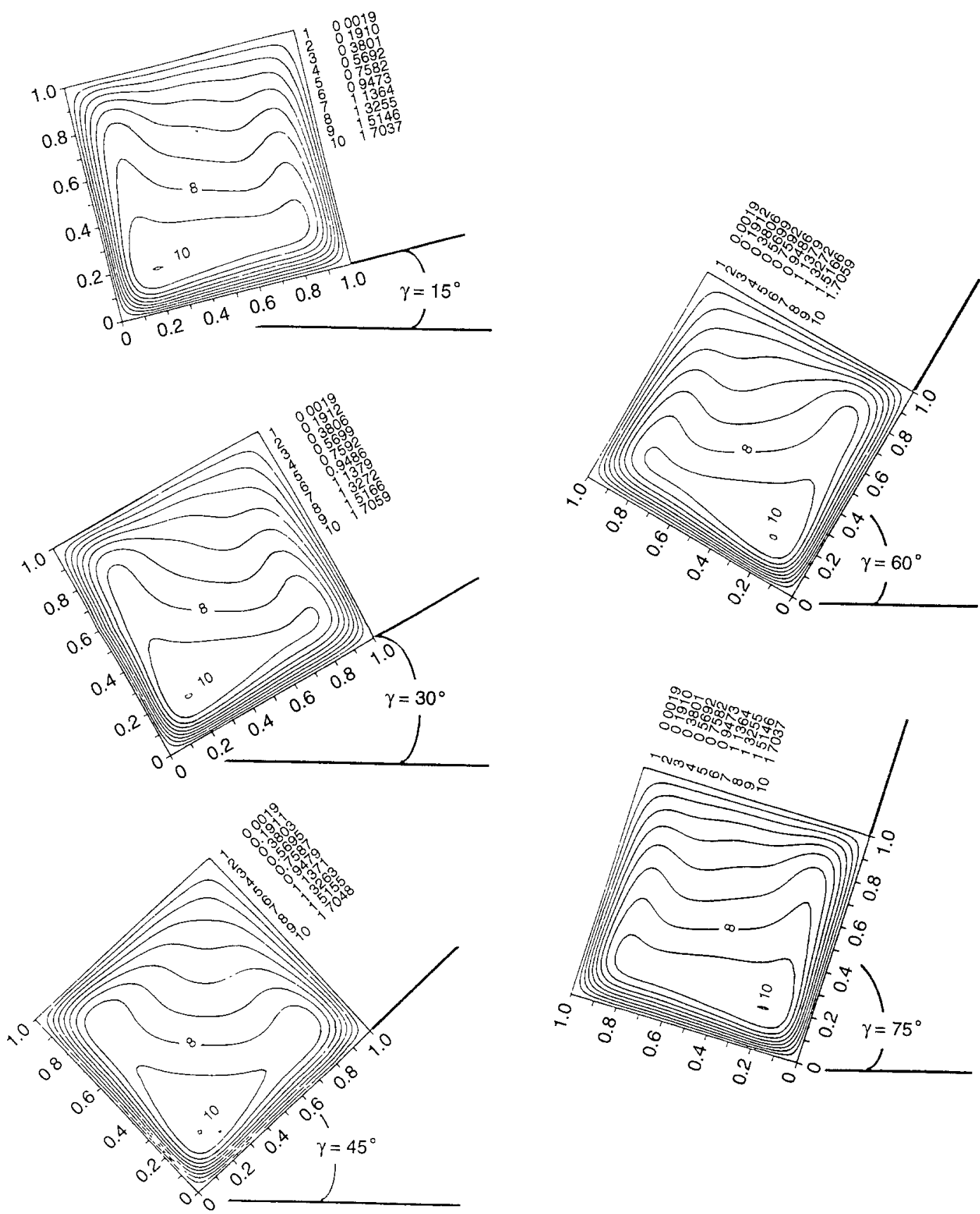

Fig. 3. Iso-lines of the axial flow velocity for flow at $R e=500, R o=0.20, \Delta T=10$, and $X / D_{h}=300$ 
The local Nusselt number $N u_{x}$ is defined as

$N u_{x}=\frac{\overline{\left(\frac{\partial \theta}{\partial n}\right)_{w}}}{\bar{\theta}}$.

Here, $n$ denotes the normal direction of wall, and $\bar{\theta}$ is the dimensionless bulk mean temperature. $\mathrm{Nu}$ is the circumferentially-averaged Nusselt number over the channel walls at each cross-section.

The ranges of the parameters used in the present study are: (1) $R e=500,1000$ and 2000, (2) $R o=0.01-0.30,(3) \zeta=1.0,(4) T_{c}=10.0$, (5) oblique angle $\gamma=15^{\circ}, 30^{\circ}, 45^{\circ}, 60^{\circ}$ and $75^{\circ}$, and (6) $P r=0.70$. The simulations cover $300 D_{h}(R e=500)$ or $600 D_{h}(R e=1000$ and 2000). The eccentricity distance is $10 D_{h}$. The axial step sizes used are $\Delta X / D_{h}=1 / 30$ for the cases of $R e=1000$ and $2000, \Delta X / D_{h}=1 / 25$ for $R e=500$. The computing times are averaged from 25,000 to 120,000 seconds on DEC Station 5000, depending upon the parameters used.
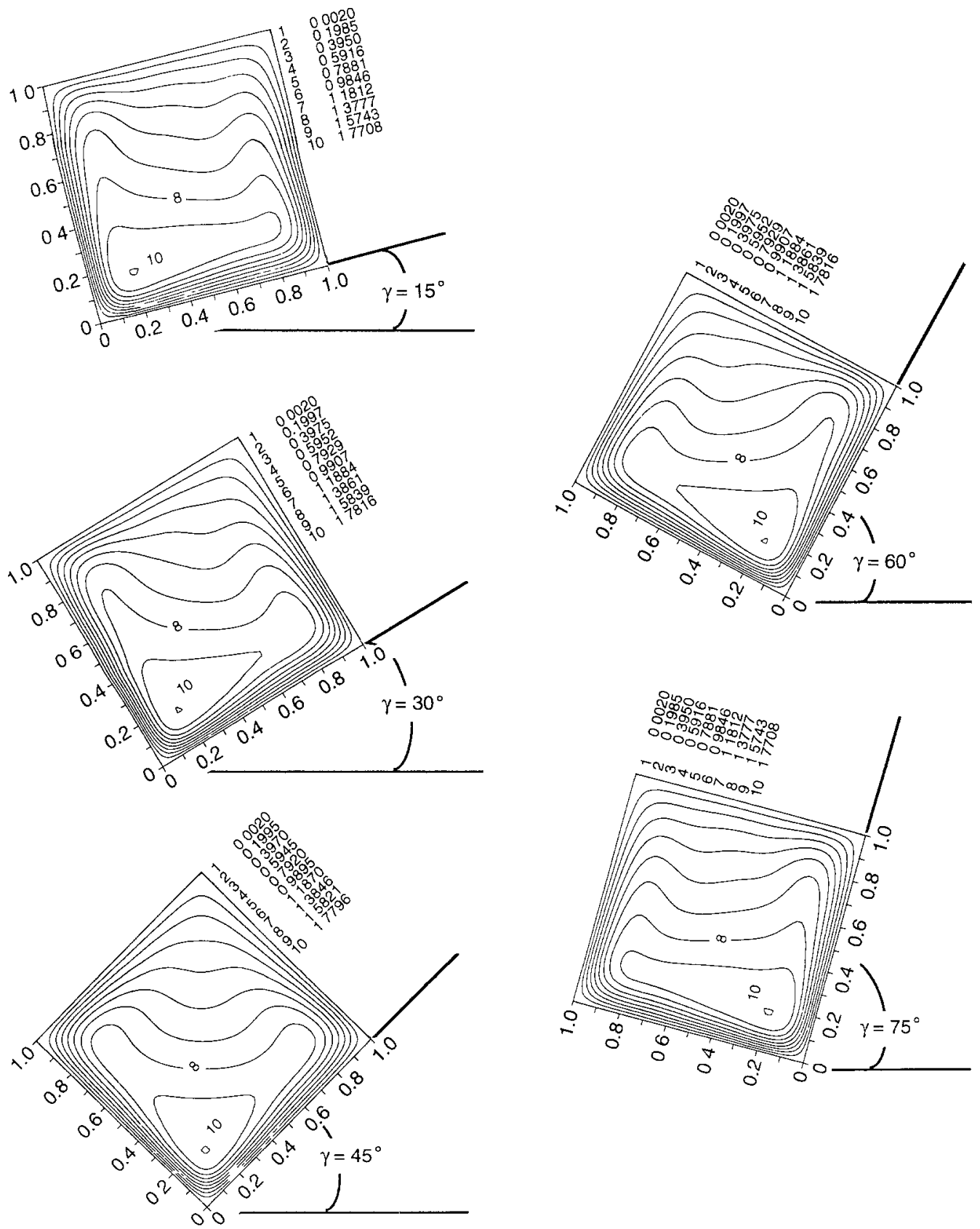

Fig. 4. Iso-lines of the axial flow velocity for flow at $R e=1000, R o=0.05, \Delta T=10$, and $X / D_{h}=600$ 


\section{3}

\section{Results and discussion}

The effects of oblique channel cross section on flow structure, and equivalently convective heat transfer inside a rotating square, isothermal channel have been numerically studied.

Figure 1 depicts an example for one period of vortex instability along the channel with zero oblique angle (the reference case). For the governing parameters of $R e=1,000$ and $R o=0.05$, the flow falls within the region of the second mode of wavy vortex type (Fann and Yang 1993b). This mode has secondary vortices (near sidewall 3) moving toward sidewalls 4 and 2, alternately. In contrast, the first mode of wavy vortex flow has secondary vortices moving toward the same sidewall (not shown). Detailed information is available in Fann and Yang (1993b).

Next is to investigate how the vortex flow structure deviates from that of the reference case, $\gamma=0^{\circ}$ with a change in the oblique angle. Results for $(R e=500, R o=0.2)$ and $(R e=1,000, R o=0.05)$ are illustrated in Figs. 3 and 4, respectively. The first significant is featured that the wavy vortex flow instability is diminished or destroyed with an introduction of the oblique angle. The second observation is the anti-symmetry of flow patterns in pairs of different oblique angles: $\gamma=15^{\circ}$ and $75^{\circ}$, and $\gamma=30^{\circ}$ and $60^{\circ}$. The third disclosure is that at $\gamma=45^{\circ}$, flow pattern is symmetrical with respect to the diagonal from the base to the tip with a clock streams, along sidewalls 4 and 3 in the right half of the channel cross section and along sidewalls 2 and 1 in the left half. Because of strong principal vortices, the tip of the axial flow velocity moves toward the base. As a matter of fact, wavy instability like the one in Fig. 2 is not completely destroyed in certain cases. For instance, wavy characteristics persists in the cases of $R e=2,000, R o=0.01$ and $\gamma=15^{\circ}$ and $30^{\circ}$, but much weaker than when $\gamma=0^{\circ}$ (not shown). The reason why obliqueness diminishes or destroys wavy instability is that is alters flow characteristics in the region near sidewall 3 . In $\gamma=0^{\circ}$ case, secondary vortices would emerge in the region along the channel centerline and near sidewall 3 (Fann and Yang 1993a and 1993b). Obliqueness reduces an

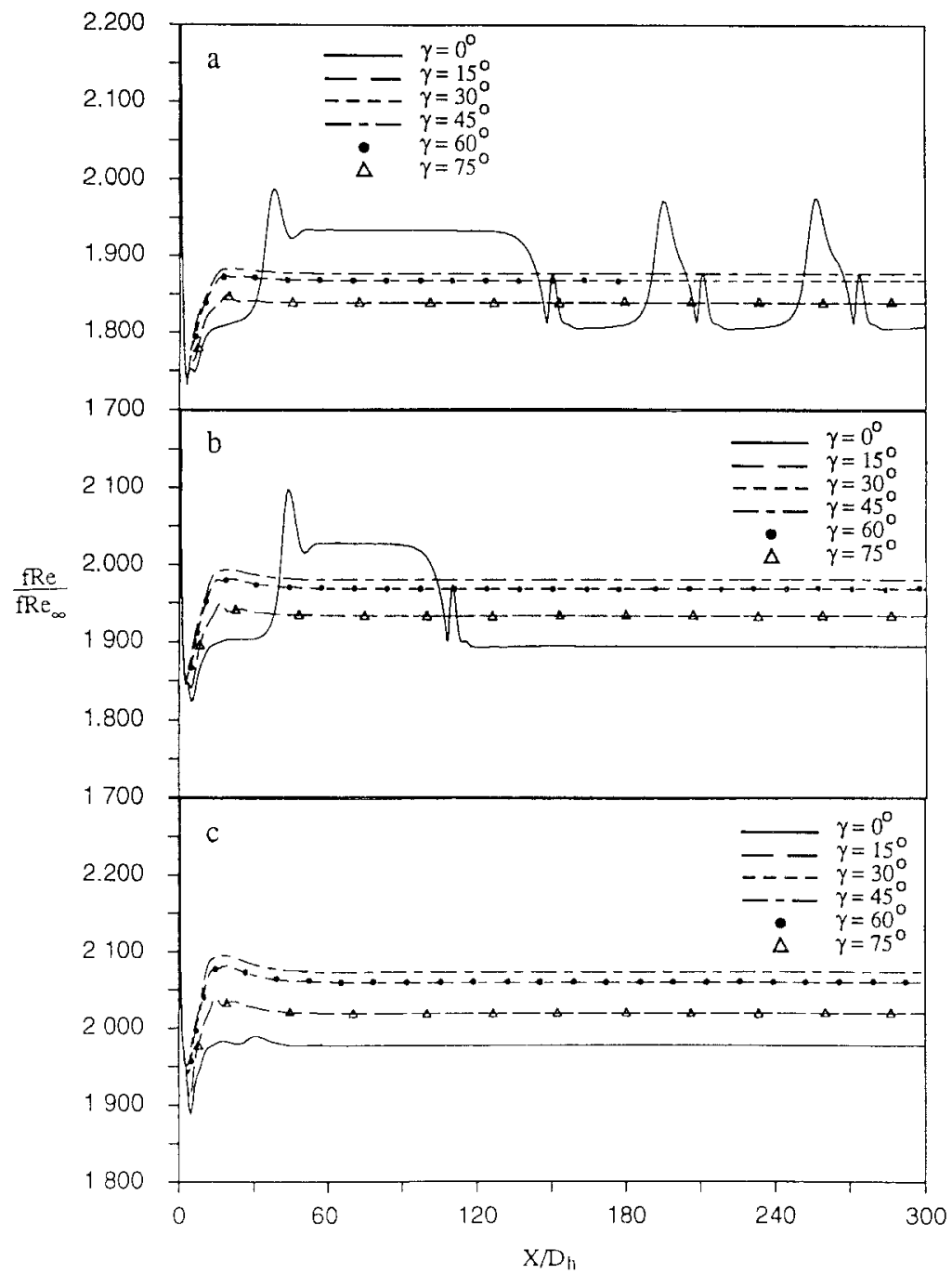

Fig. 5. Friction factor distributions along the channel for flows at $R e=500, \Delta T=10$, and (a) $R o=0.20$, (b) $R o=0.25$, (c) $R o=0.30$ 

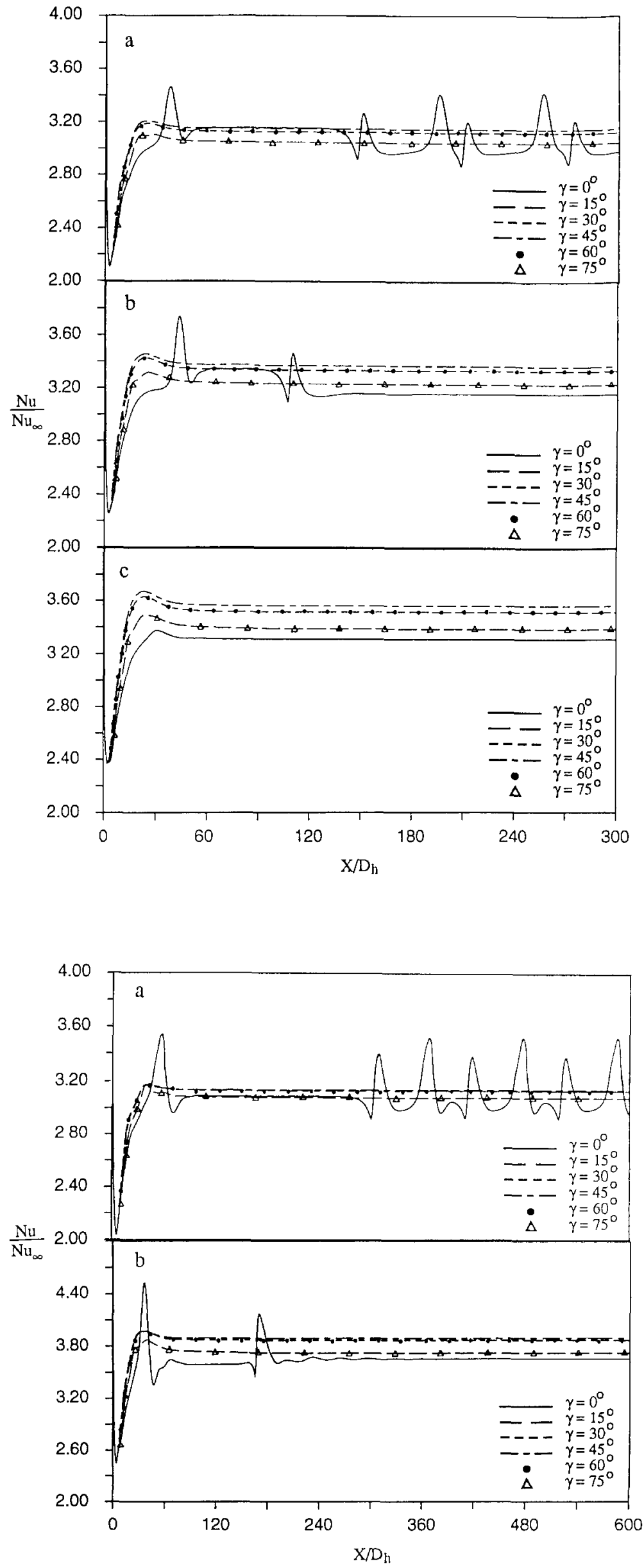

Fig. 6. Circumferentially-averaged Nusselt number distributions along the channel for flows at $R e=500, \Delta T=10$, and (a) $R o=0.20$, (b) $R o=0.25$, (c) $R o=0.30$
Fig. 7. Circumferentiallyaveraged Nusselt number distributions along the channel for flows at $R e=1000, \Delta T=10$, and (a) $R o=0.050$, (b) $R o=0.10$ 
opportunity of holding mass in that region and consequently the principal vortices prevail throughout the channel cross section, making it difficult for a small circulation to emerge.

Figure 5 depicts the distribution of friction factors along the channels of different oblique angles with a flow of $R e=500$. A symmetry in flow patterns for $\gamma=15^{\circ}$ and $75^{\circ}$, and for $\gamma=30^{\circ}$ and $60^{\circ}$ results in the same values of $f R e$. In Figs. 5(a) and $5(\mathrm{~b})$, the influence of secondary vortices disappear in the presence of obliqueness. It is revealed that except the $\gamma=0^{3}$ case characterized by secondary vortices, the friction factor is enhanced with an increase in, reaching the highest distribution at $\gamma=45^{\circ}$ followed by a decrease with a further increase in the oblique angle. The rate of increase in $f R e$ is retarded as $\gamma$ approaches $45^{\circ}$ where the vortex flow achieve a maximum strength.

The influence of flow characteristics is also reflected in heat transfer performance. Figures 6 and $7 \mathrm{~s}^{\star}$ how the circumferentially-averaged Nusselt number distributions at $R e=500$ and 1,000 , respectively. It is observed in Figs. $7(\mathrm{a})$ and (b) that even in the presence of secondary vortices, the heat transfer performance may be inferior than those in the channels with obliqueness. It is also found that the extent of heat transfer enhancement from $\gamma=30^{\circ}$ to $45^{\circ}$ can be very small in those cases with a high Reynolds number and a low Rossby number. Figures 8 and 9 show the local heat transfer characteristics along the channels of $R e=1,000$. In the $\gamma=0^{\circ}$ case, sidewall 1 is the leading wall with sidewall 3 as the trailing wall. It is well known in such case that heat transfer wall is better than that from the leading wall at $\gamma=0^{\circ}$. Figure 8 depicts that the difference in heat transfer performance between the trailing and leading walls gradually diminishes with an increase in obliqueness. The reason is that as the oblique angle appoaches $75^{\circ}$, the role of sidewalls 1 and 3 become more and more like that of sidewalls 2 and 4 in the $\gamma=0^{\circ}$ case. In the absence of the wavy instability, sidewalls 2 and 4 of non-oblique channels have an identical heat transfer performance. It is also revealed in Fig. 8 that the highest heat transfer performance at sidewall 3 does not occur at $\gamma=15^{\circ}$, but at $\gamma=30^{\circ}$ with sidewall 1 having the worst performance at $\gamma=15^{\circ}$. Figure 9 illustrates different local heat transfer characteristics at sidewalls 2 and 4. It is found that the difference between the two walls is enhanced with an increase in. The best performance on sidewall 2 occurs at $\gamma=60^{\circ}$, while the worst on sidewall 4 is at $\gamma=75^{\circ}$.

Figure 10 illustrates changes of the overall heat transfer performance, in term of the Nusselt number averaged over the computed channel length, $\overline{N u}$ with the oblique angle. It is seen that an oblique angle of $45^{\circ}$ provides the best performance. $\overline{N u}$ enhances with the Rossby number under the same flow condition, $R e$. It was featured in Fann and Yang (1993b) that heating exerts a great influence on vortex flow structure and consequently heat transfer performance. Figures 11 through 14 show heating effects for $\gamma=0^{\circ}$ case. It is seen that an increase in the wall heating (i.e. $\Delta T$ ) enhances heat transfer only near the entrance

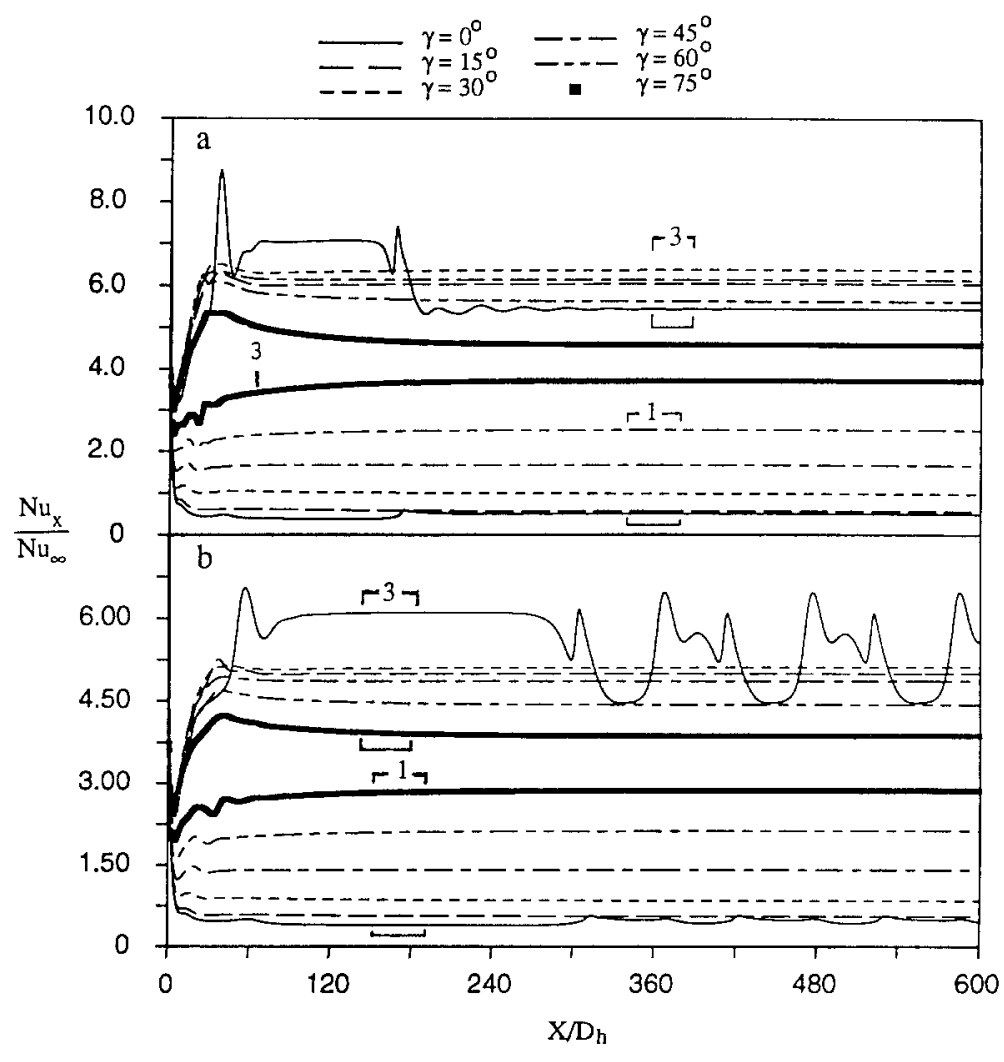

Fig. 8. Local Nusselt number distributions on the Sidewalls No. 1 and No. 3 along the channel for flows at $R e=1000, \Delta T=10$, and (a) $R o=0.10$, (b) $R o=0.050$ 


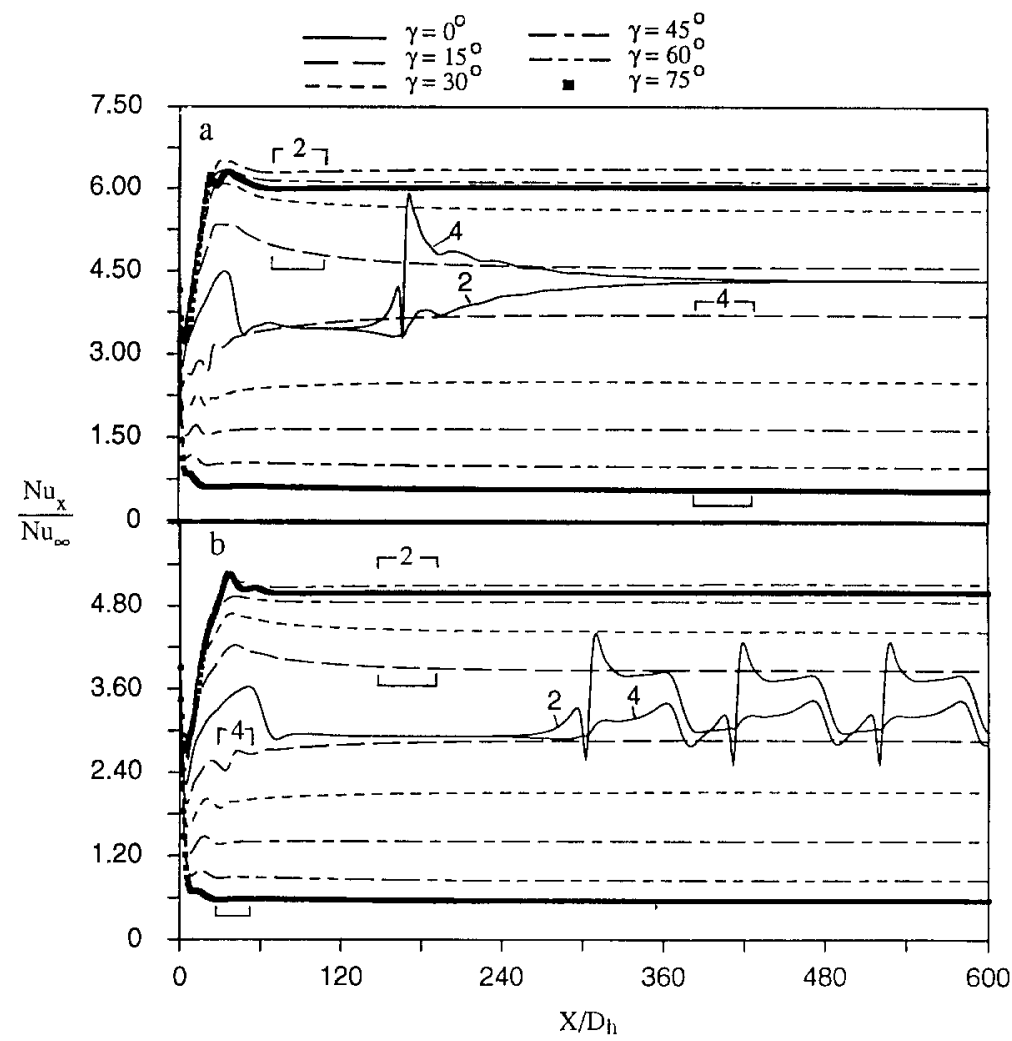

Fig. 9. Local Nusselt number distributions on the Sidewalls No. 2 and No. 4 along the channel for flows at $R e=1000, \Delta T=10$, and (a) $R o=0.10$, (b) $R o=0.050$

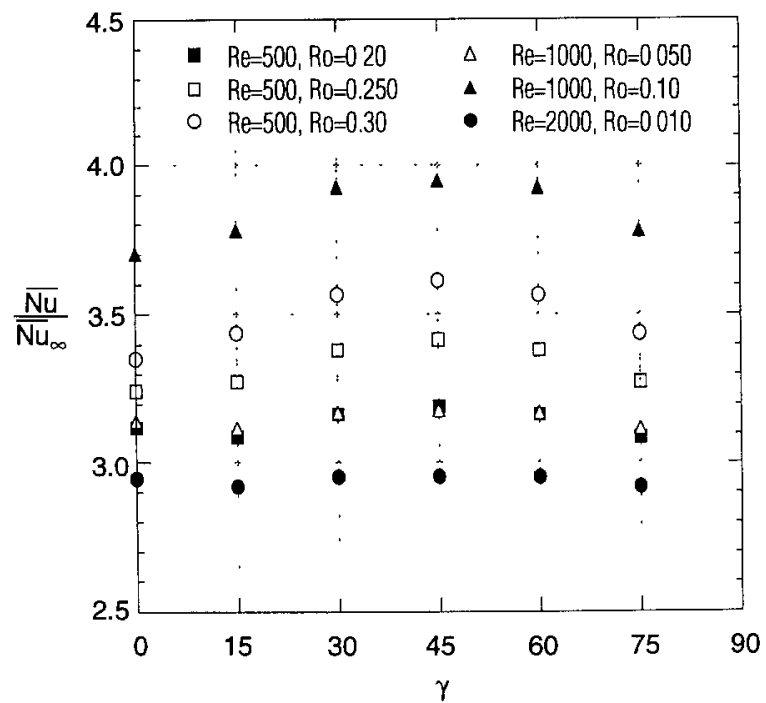

but exerts practically no influence further downstream. This is because wavy instability and secondary vortices can hardly survive in a non-oblique channel, with the flow characteristics remaining unchanged except in the entrance region where the flow is developing and changing drastically. Also revealed is that the local heat transfer at sidewalls 2 and 3 enhance with wall heating. The opposite is true for sidewalls 1 and 4 .

Equations (2) and (3) can explain how an increase in wall heating affects heat transfer: In the entrance region, and increase in the Grashof number $(G r)$ resulting from an increase in $\Delta T$ can cause a drastic change in $\zeta$ because of drastic variation in $\partial \theta / \partial y$ and $\partial \theta / \partial z$, and $\theta$ there. As the flow proceeds downstream, the fluid temperature gradually approaches an isothermal status and, $\theta$ and $\partial \theta / \partial y$ and $\partial \theta / \hat{c} z$ becomes too small to affect the flow and heat transfer performance. 


\section{4}

\section{Conclusions}

A computational method has been used to investigate the effects of obliqueness on the flow structure and heat transfer performance inside an isothermal, rotating square channel. The oblique angle, wall heating, flow rate and rotating speed have been varied. The following conclusions have been derived from the study:

(i) Obliqueness, $\gamma$, supresses or destroys secondary vortices and wavy instability in the channel cross sections.

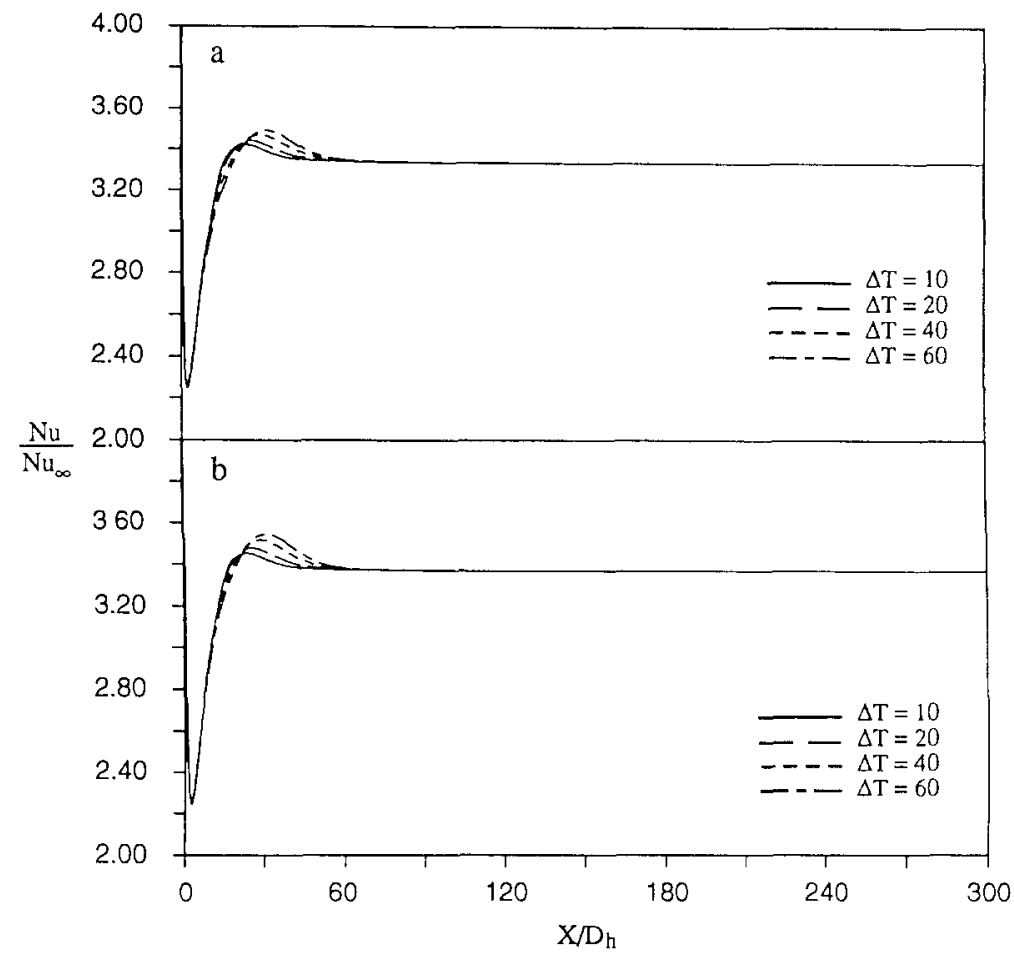

11

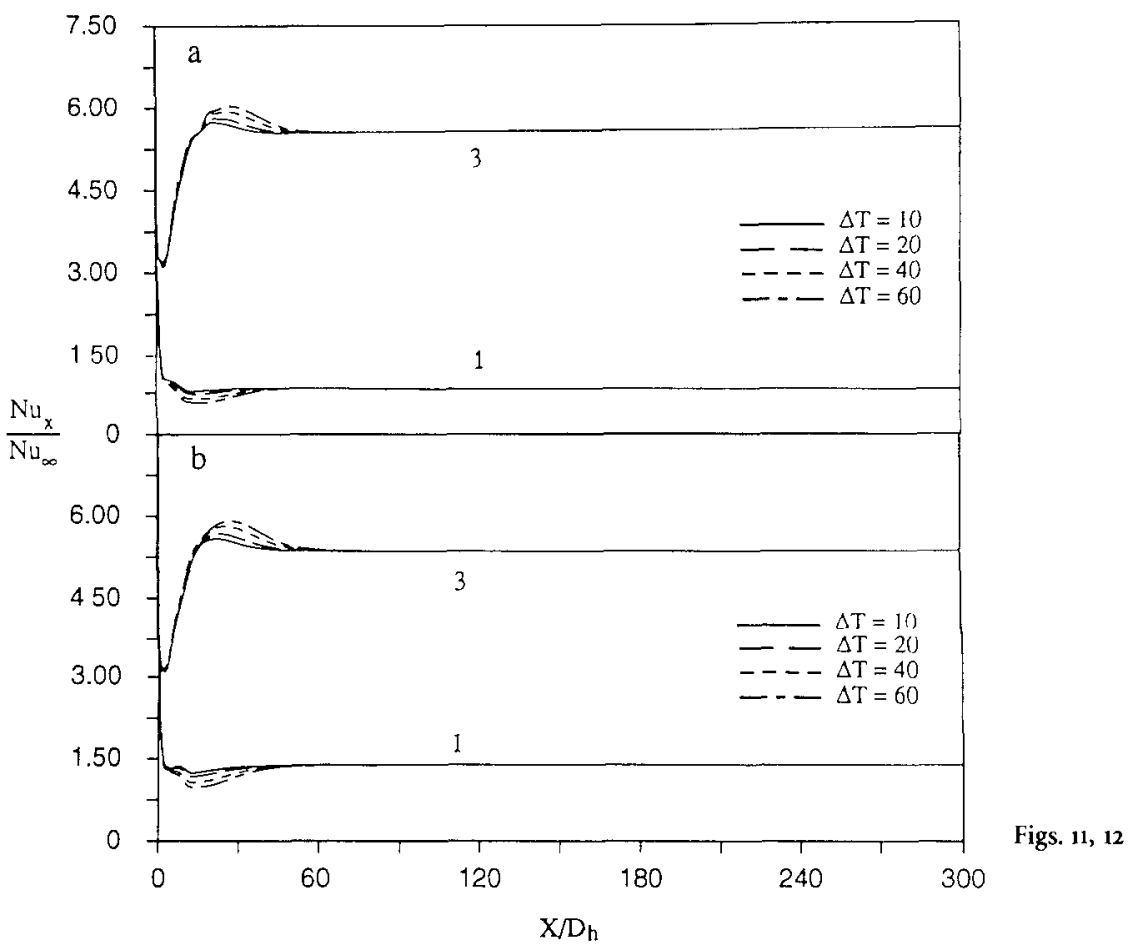




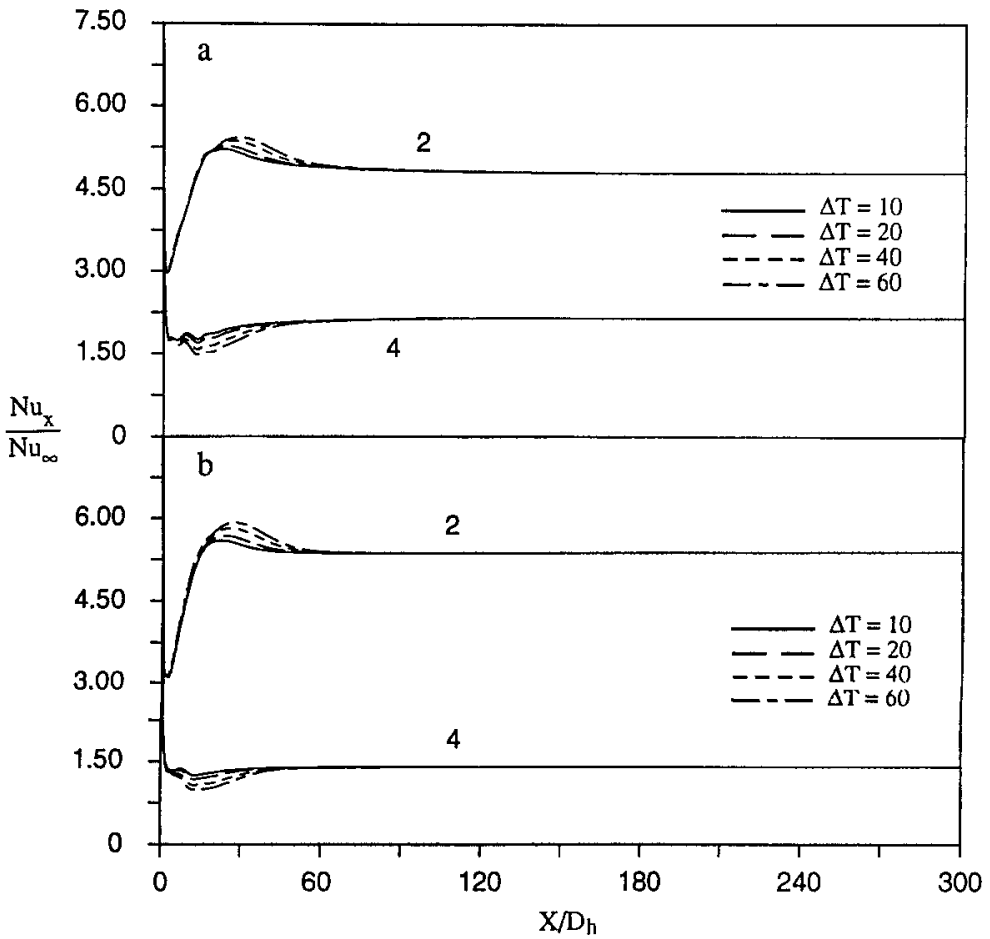

13

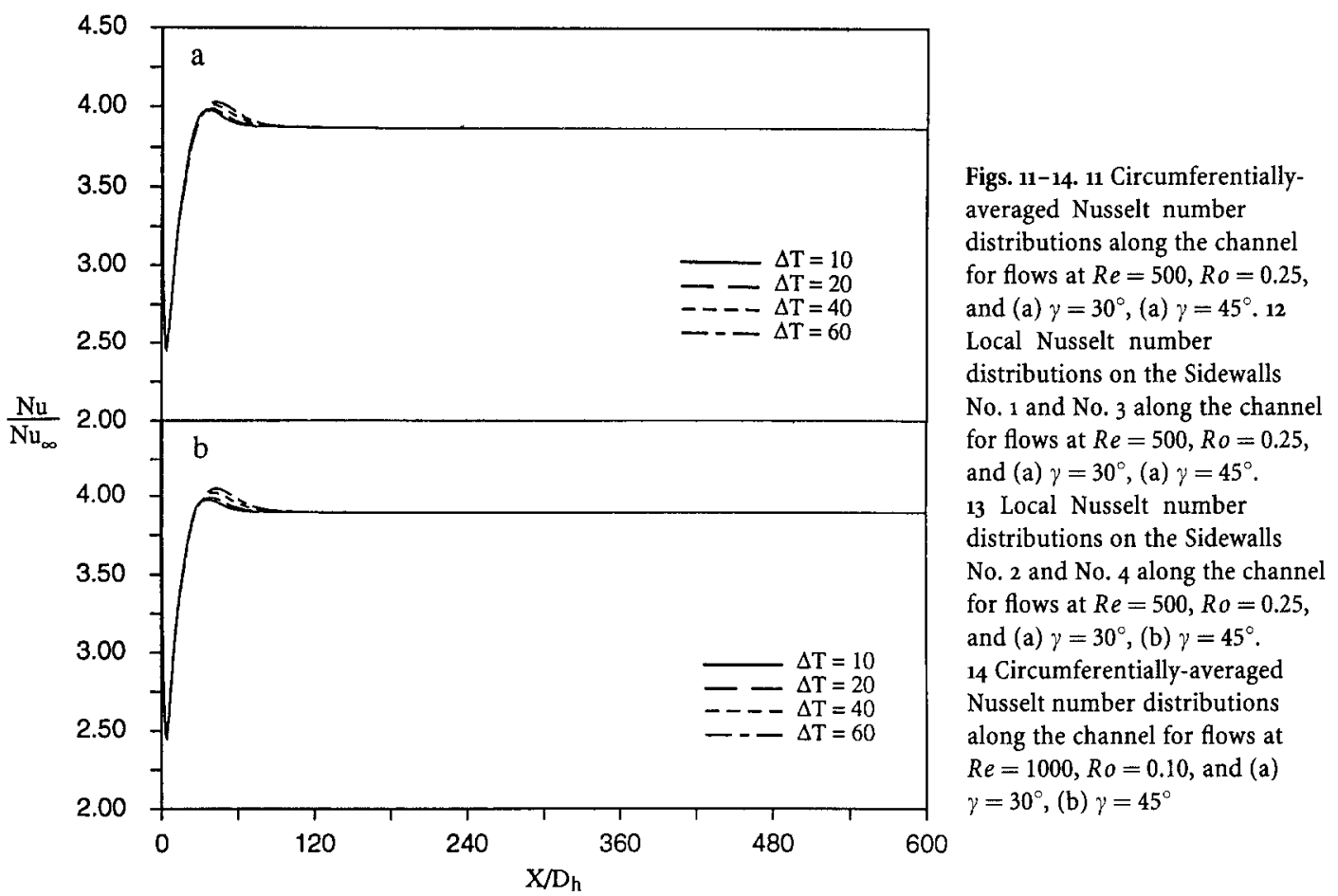

14

(ii) In turn, it enhances the overall heat transfer performance, with the best at $\gamma=45^{\circ}$.

(iii) Two oblique angles in a pair, including $\left(\gamma=15^{\circ}\right.$ and $\left.75^{\circ}\right)$ and $\gamma=30^{\circ}$ and $\left.60^{\circ}\right)$, form a symmetric flow structure and consequently share identical distributions in the friction factor and circumferentially-averaged Nusselt number.

(iv) An increase in wall heating enhances heat transfer only near the channel entrance but not further downstream. It results in an augmentation of the local Nusselt number distributions on sidewalls 2 and 3 but a reduction on sidewalls 1 and 4 . 


\section{References}

Alfredsson, P. H.; Persson, H. 1989: Instability in channel flow with system rotation. J. Fluid Mech. 202: 543-557

Fann, S.; Yang, W.-J. 1992: Hydrodynamically-thermally developing laminar flow through rotating channels having isothermal walls. Numerical Heat Transfer 22(3): 257-288

Fann, S.; Yang, W.-J.; Mochizuki, S. 1992: Heat and fluid flow at entrance regions of rotating iso-heat flux channels with laminar through flow. Inter. J. Num. Meth. Heat Fluid Flow 2: 335-358

Fann, S.; Yang, W.-J. 1993a: A numerical study of developing radial flow with rotation. Int. J. Num. Meth. Heat Fluid Flow (in press)

Fann, S.; Yang, W.-J. 1993b: Heating effects on laminar flow through a rotating square channel. AIAA J. Thermophysics Heat Transfer (in press)

Finlay, W. H. 1990: Transition to oscillatory motion on rotating channel flow. J. Fluid Mech. 215: 209-227

Finlay, W. H. 1992: Transition to turbulence in a rotating channel. J. Fluid Mech. 237: 73-99

Harasgama, S. P.; Morris, W. D. 1988: The influence of rotation on the heat transfer characteristics of circular, triangular, and square-section coolant passages of gas turbine rotor blade. ASME J. Turbomachinery 110: 44-50

Hart, J. E. 1971: Instability and secondary motion in a rotating channel flow. J. Fluid Mech. 45(2): 341-351

Hwang, G. J.; Jen, T. C. 1990: Convective heat transfer in rotating isothermal ducts. Inter. J. Heat and Mass Transfer 33 (9): $1817-1828$

Jen, T. C.; Lavine, A. S.; Hwang, G. L. 1992: Simultaneously developing laminar convection in rotating isothermal square channels. Inter. J. Heat Mass Transfer 35(1): 239-254

Jen, T. C.; Lavine, A. S. 1992: Laminar heat transfer and fluid flow in the entrance region of a rotating duct with rectangular cross section: the effect of aspect ratio. ASME J. Heat Transfer 114: 574-581

Johnston, J. P.; Halleen, R. M.; Lezius, D. K. 1972: Effects of spanwise rotation on the structure of two-dimensional fully developed turbulent channel flow. J. Fluid Mech. 56(3): 533-557

Kheshgi, H. S.; Scriven, L. E. 1985: Viscous flow through a rotating square channel. Physics of Fluids 28(10): 2968-2979 Kuz'minskii, L. V.; Smirnov, E. M.; Yurkin, S. V. 1983: Longitudinal cellular structure of Taylor-Gortler vortices on the highpressure side of rotating channels. J. Appl. Mech. Tech. Physics 24: 882-886

Lezius, D. K.; Johnston, J. P. 1976: Roll-cell instabilities in rotating laminar and turbulent channel flow. J. Fluid Mech. 77: 153-175 Morris, W. D.; Ghavami-Nasr, G. 1991: Heat transfer mesurements in rectangular channels with orthogonal mode rotation. ASME J. Turbomachinery 113: 339-345

Ramakrishna, K.; Rubin, S. G.; Khosla, P. K. 1982: Laminar natural convection along vertical square ducts. Numerical Heat Transfer 5: 59-79

Siegel, R. 1985: Analysis of buoyancy effect on fully developed laminar heat transfer in a rotating tube. ASME J. Heat Transfer 107: $338-344$

Smirnov, E. M.; Yurkin, S. V. 1983: Fluid flow in a rotating channel of square section. Fluid Dynamics 18: 850-855

Speziale, C. G. 1982: Numerical study of viscous flow in rotating rectangular ducts. J. Fluid Mech. 122: 251-271

Speziale, C. G.; Thangam, S. 1983: Numerical study of secondary flows and roll-cell instability in rotating channel flow. J.

Fluid Mech. 130: 377-395 